\title{
Locally acquired severe non-Ol and non-O139 Vibrio cholerae infection associated with ingestion of imported seafood
}

To THE EDITOR: We report a case of severe Vibrio cholerae infection acquired in Sydney, likely due to ingestion of imported seafood.

An 83-year-old man with Parkinson disease presented with a 3-day history of vomiting, largevolume watery diarrhoea and acute renal impairment necessitating admission to the intensive care unit. Blood cultures grew curved gramnegative bacilli, and intravenous piperacillin-clavulanic acid was commenced. Subsequent microbiological testing of blood isolated $V$. cholerae, prompting testing and confirmation of $V$. cholerae in stool cultures. The strain was identified as non-O1 and non-O139 by serotyping, and toxin gene-negative by polymerase chain reaction testing.

The patient subsequently reported ingestion of imported seafood (a marinara mix containing mussels from Chile, prawns from Vietnam and squid from China), purchased from a local supermarket, although none of the suspected food was available for testing. He had no recent travel history or exposure to marine or brackish-water environments and no unwell contacts.

Antimicrobial therapy was changed to ciprofloxacin. The patient's recovery was complicated by caecal pseudoobstruction requiring endoscopic decompression. He was discharged after 2 weeks of antibiotic treatment.

Although rare, sporadic cases of both epidemic (O1 and O139 
serotypes) and non-epidemic (non-O1 and non-O139 serotypes) $V$. cholerae infection have been reported in Australia. ${ }^{1}$ Australian cases have been linked to ingestion of imported seafood, with a notable outbreak in Sydney associated with imported whitebait. ${ }^{2} V$. cholerae is known to be present in Australian estuaries, and some endemic cases have been associated with local aquatic exposure. $^{3}$

While only $\mathrm{O} 1$ and $\mathrm{O} 139$ isolates are mandated for reporting to Australian public health units, non-epidemic strains of $V$. cholerae are associated with bacteraemia and a poor prognosis. ${ }^{4}$ Clinically suspected cases of $V$. cholerae infection should be reported to public health units, pending microbiological confirmation. As identification of Vibrio species is not routinely done on stool cultures, a suspicion of Vibrio infection must be communicated to laboratories.

Under current Australian law, only imported cooked prawns are required to be tested for Vibrio species contamination, and there is no restriction on the geographical origin of seafood imported into Australia. 5,6 This case highlights an ongoing risk of potentially severe $V$. cholerae infection from imported seafood, and it should be considered as a differential diagnosis for patients presenting with severe enteritis and a compatible exposure history.

Chiao-Yun Hsu Medical Registrar'

Simon Pollett Infectious Diseases Advanced Trainee ${ }^{2}$

Patricia Ferguson Infectious Diseases Physician ${ }^{2}$

Brenden J McMullan Microbiology Registrar ${ }^{2}$

Vicki Sheppeard Public Health Physician ${ }^{3}$

Suzanne E Mahady Epidemiologist ${ }^{4}$

1 Westmead Hospital, Sydney, NSW.

2 Centre for Infectious Diseases and Microbiology, Westmead Millennium Institute for Medical Research, Sydney, NSW.

3 Public Health Unit, Nepean Blue Mountains and Western Sydney Local Health Districts, Sydney, NSW.

4 Sydney School of Public Health, University of Sydney, Sydney, NSW.

julia.cy.hsu@gmail.com

Competing interests: No relevant disclosures.

doi: 10.5694/mjal3.10087

1 Rogers RC, Cuffe RG, Cossins YM, et al. The Queensland cholera incident of 1977.2. The epidemiological investigation. Bull World Health Organ 1980; 58: 665-669.

2 Forssman B, Mannes T, Musto J, et al. Vibrio cholerae $\mathrm{Ol}$ El Tor cluster in Sydney linked to imported whitebait. Med J Aust 2007; 187: 345-347.
3 Desmarchelier PM, Wong FY, Mallard K. An epidemiological study of Vibrio cholerae $\mathrm{Ol}$ in the Australian environment based on rRNA gene polymorphisms. Epidemiol Infect 1995; 115: 435-446.

4 Ko WC, Chuang YC, Huang GC, Hsu SY. Infections due to non-Ol Vibrio cholerae in southern Taiwan: predominance in cirrhotic patients. Clin Infect Dis 1998; 27: 774-780.

5 Food Standards Australia New Zealand. Safe seafood Australia: a guide to the Australian Primary Production and Processing Standard for Seafood. Canberra: FSANZ, 2006.

6 Australian Government Department of Agriculture, Fisheries and Forestry. Imported food notice 09/12. Tests applied to risk category foods. Canberra: DAFF, 2012. http://www.daff.gov.au/ aqis/import/food/notices/2009/2012/ifn-0912 (accessed Sep 2012). 